\title{
Relations between anisotropic Besov spaces and multivariate Bernstein-Durrmeyer operators
}

Guo Feng ${ }^{1,2^{*}}$ and Yuan Feng ${ }^{3}$

\section{"Correspondence:} gfeng@tzc.edu.cn

${ }^{1}$ School of Mathematics and Information Engineering, Taizhou University, Taizhou, Zhejiang 317000, China

${ }^{2}$ School of Mathematical Sciences, Beijing Normal University, Beijing, 100875, China

Full list of author information is

available at the end of the article

\begin{abstract}
In this paper, we use the multivariate Bernstein-Durrmeyer operators defined on the simplex to characterize anisotropic Besov spaces.
\end{abstract}

MSC: $41 \mathrm{~A} 27 ; 41 \mathrm{~A} 36$

Keywords: multivariate Bernstein-Durrmeyer operators; K-functional; anisotropic Besov spaces

\section{Introduction and some notations}

Let $\mathrm{T}$ be the simplex in $\mathbb{R}^{d}$ defined by

$$
\mathrm{T}=\left\{\mathrm{x}=\left(x_{1}, x_{2}, \ldots, x_{d}\right): x_{i} \geq 0, i=1,2, \ldots, d,|\mathrm{x}|=\sum_{i=1}^{d} x_{i} \leq 1\right\}
$$

Let $L_{p}(\mathrm{~T}):=L_{\mathrm{p}}(\mathrm{T}), \mathrm{p}=\left(p_{1}, p_{2}, \ldots, p_{d}\right), p_{1}=p_{2}=\cdots=p_{d}=p, 1 \leq p<\infty$, be the space consisting of all Lebesgue measurable functions $f$ on $\mathrm{T}$ for which the norm $\|f\|_{p}:=$ $\left(\int_{\mathrm{T}}|f(\mathrm{x})|^{p} d x\right)^{1 / p}$ is finite. Let $C(\mathrm{~T}):=L_{\infty}(\mathrm{T}), \infty=(\infty, \infty, \ldots, \infty)$ be the space consisting of all continuous functions $f$ on $\mathrm{T}$ for which the norm $\max _{\mathrm{x} \in \mathrm{T}}|f(\mathrm{x})|$ is finite.

Let $f \in L_{1}(\mathrm{~T})$. For each $n \in \mathbb{N}$, the multivariate Bernstein-Durrmeyer operators of $f$ are defined by [1]

$$
M_{n, \mathrm{~d}}(f ; \mathrm{x})=\sum_{|\mathrm{k}| \leq n} p_{n, \mathrm{k}}(\mathrm{x}) \frac{(n+d) !}{n !} \int_{\mathrm{T}} p_{n, \mathrm{k}}(\mathrm{u}) f(\mathrm{u}) d \mathrm{u},
$$

where

$$
p_{n, \mathrm{k}}(\mathrm{x})=\frac{n !}{\mathrm{k} !(n-|\mathrm{k}|) !} \mathrm{x}^{\mathrm{k}}(1-\mathrm{x})^{n-|\mathrm{k}|}, \quad \mathrm{x} \in \mathrm{T},
$$

$\mathrm{x}=\left(x_{1}, x_{2}, \ldots, x_{d}\right) \in \mathbb{R}^{d}, \mathrm{k}=\left(k_{1}, k_{2}, \ldots, k_{d}\right) \in \mathbb{N}_{0}^{d}, \mathbb{N}_{0}^{d}=\overbrace{\mathbb{N}_{0} \times \mathbb{N}_{0} \times \cdots \times \mathbb{N}_{0}}^{d}, \mathbb{N}_{0}=\mathbb{N} \cup\{0\}$, $|x|=\sum_{i=1}^{d} x_{i}, \mathrm{x}^{\mathrm{k}}=x_{1}^{k_{1}} x_{2}^{k_{2}} \cdots \mathrm{x}_{d}^{k_{d}},|\mathrm{k}|=\sum_{i=1}^{d} k_{i}, \mathrm{k} !=k_{1} ! k_{2} ! \cdots k_{d} !$

( 2013 Feng and Feng; licensee Springer. This is an Open Access article distributed under the terms of the Creative Commons Attribution License (http://creativecommons.org/licenses/by/2.0), which permits unrestricted use, distribution, and reproduction in any medium, provided the original work is properly cited. 
For $\mathrm{x} \in \mathrm{T}$, we denote

$$
\varphi_{i j}^{2}(\mathrm{x})= \begin{cases}x_{i}(1-|\mathrm{x}|) & \text { for } i=j=1,2, \ldots, d \\ x_{i} x_{j} & \text { for } 1 \leq i<j \leq d\end{cases}
$$

Let $D_{i}=D_{i i}=\frac{\partial}{\partial x_{i}}, 1 \leq i \leq d ; D_{i j}=D_{i}-D_{j}, 1 \leq i<j \leq d ; D^{k}=D_{1}^{k_{1}} D_{2}^{k_{2}} \cdots D_{d}^{k_{d}}, \mathrm{k} \in N_{0}^{d}$, and

$$
D_{i j}^{2}(\mathrm{x})= \begin{cases}\frac{\partial^{2}}{\partial x_{i}^{2}} & \text { for } i=j=1,2, \ldots, d, \\ \left(\frac{\partial}{\partial x_{i}}-\frac{\partial}{\partial x_{j}}\right)^{2} & \text { for } 1 \leq i<j \leq d .\end{cases}
$$

Definition 1.1 Let $L_{p}:=L_{p}(\mathrm{~T}), 1 \leq p<\infty$, and weighted Sobolev spaces are given by

$$
W_{\Phi, p}^{2}=\left\{g\left|g \in L_{p}, D^{k} g,\right| k \mid \leq 2 \text { are in } L_{\mathrm{loc}}(\stackrel{\mathrm{T}}{\mathrm{T}}) \text {, and } \varphi_{i j}^{2} D_{i j}^{2} f \in L_{p}, 1 \leq i \leq j \leq d\right\} \text {, }
$$

where the derivatives are in the sense of distributions, and $\stackrel{\circ}{\mathrm{T}}$ is the interior of $\mathrm{T}$.

The K-functional of Ditzian-Totik type is given by

$$
K_{\varphi}^{2}\left(f ; t_{l}^{2}\right)_{p}=\inf _{g \in W_{\Phi, p}^{2}}\left\{\|f-g\|_{p}+t_{l}^{2} \Phi(g)_{p}\right\}, \quad t_{l}>0, l=1,2, \ldots, d
$$

where $\mathrm{t}=\left(t_{1}, t_{2}, \ldots, t_{d}\right), \Phi(g)_{p}:=\|g\|_{p}+\sum_{1 \leq i \leq j \leq d}\left\|\varphi_{i j}^{2} D_{i j}^{2} g\right\|_{p}$.

The anisotropic Besov spaces [2] are given by

$$
B_{p, q}^{\frac{\theta}{2}}:=\left(L_{p}, W_{\Phi, p}^{2}\right)_{\frac{\theta}{2}, q}
$$

where $\theta=\left(\theta_{1}, \theta_{2}, \ldots, \theta_{d}\right), 1 \leq p, q<\infty, n \in \mathbb{N}, n>2>\theta_{l}>0$.

By [3] and the definition of anisotropic Besov spaces, it is not difficult to get the following.

Theorem 1.2 Suppose $1 \leq p, q<\infty, n \in \mathbb{N}, n>2>\theta_{l}>0, l=1,2, \ldots, d$. Then

$$
f \in B_{p, q}^{\frac{\theta}{2}} \Leftrightarrow \int_{0}^{\infty}\left[t_{l}^{-\frac{\theta_{l}}{2}} K_{\varphi}^{2}\left(f ; t_{l}^{2}\right)_{p}\right]^{q} \frac{d t_{l}}{t_{l}}<\infty
$$

and

$$
\int_{0}^{\infty}\left[t_{l}^{-\frac{\theta_{l}}{2}} K_{\varphi}^{2}\left(f ; t_{l}^{2}\right)_{p}\right]^{q} \frac{d t_{l}}{t_{l}}<\infty \Leftrightarrow \int_{0}^{1}\left[t_{l}^{-\frac{\theta_{l}}{2}} K_{\varphi}^{2}\left(f ; t_{l}^{2}\right)_{p}\right]^{q} \frac{d t_{l}}{t_{l}}<\infty .
$$

In this paper, we use the multivariate Bernstein-Durrmeyer operators defined on the simplex to characterize anisotropic Besov spaces. We will show, for $1 \leq p, q<\infty, n \in \mathbb{N}$, $n>2>\theta_{l}>0$, that

$$
f \in B_{p, q}^{\frac{\theta}{2}} \quad \Leftrightarrow \quad\left\{\sum_{n=1}^{\infty}\left[n^{\frac{\theta_{l}}{2}}\left\|L_{n}(f)-f\right\|_{p}\right]^{q} \frac{1}{n}\right\}^{\frac{1}{q}}<\infty
$$

For convenience, throughout this paper, $M$ denotes a positive constant independent of $x, n$ and $f$ which may be different in different places. 


\section{Auxiliary lemmas}

To prove the theorems, we need the following lemmas. The following two lemmas were proved in [4].

Lemma 2.1 If $1 \leq p<\infty, f \in L_{p}, n \in \mathbb{N}$, then

$$
\begin{aligned}
& \left\|M_{n, d}(f)\right\|_{p} \leq M\|f\|_{p}, \\
& \left\|\varphi_{i j}^{2} D_{i j}^{2} M_{n, d}(f)\right\|_{p} \leq M n\|f\|_{p}, \quad 1 \leq i \leq j \leq d .
\end{aligned}
$$

Lemma 2.2 If $1 \leq p<\infty, f \in W_{\Phi, p}^{2}, n \in \mathbb{N}, n>2$, then

$$
\left\|\varphi_{i j}^{2} D_{i j}^{2} M_{n, d}(f)\right\|_{p} \leq M\left\|\varphi_{i j}^{2} D_{i j}^{2} f\right\|_{p}, \quad i=1,2, \ldots, d .
$$

Lemma 2.3 Suppose $1 \leq p<\infty, f \in L_{p}, n \in \mathbb{N}, n>2$. Then

$$
\left\|M_{n, d}(f)-f\right\|_{p} \leq M K_{\varphi}^{2}\left(f ; n^{-1}\right)_{p} .
$$

Proof Let $f \in L_{p}$, It is shown in [5] that there exists a constant $M>0$ such that

$$
M^{-1} \omega_{\varphi}^{2}\left(f ; t_{l}\right)_{p} \leq K_{\varphi}^{*, 2}\left(f ; t_{l}^{2}\right)_{p} \leq M \omega_{\varphi}^{2}\left(f ; t_{l}\right)_{p},
$$

where $\omega_{\varphi}^{2}\left(f ; t_{l}\right)_{p}$ is the modulus of smoothness of Ditzian-Totik type defined by

$$
\begin{aligned}
& \omega_{\varphi}^{2}\left(f ; t_{l}\right)_{p}:=\sup _{0 \leq h \leq t_{l}} \sum_{1 \leq i \leq j \leq d}\left\|\Delta_{h \varphi_{i j} e_{i j}}^{2} f\right\|_{p}, \quad t_{l}>0, l=1,2, \ldots, d, \\
& \left\|\Delta_{h e}^{2} f(\mathrm{x})\right\|_{p}= \begin{cases}f\left(\mathrm{x}+\frac{h e}{2}\right)-2 f\left(\mathrm{x}+\frac{h e}{2}\right)+f\left(\mathrm{x}-\frac{h e}{2}\right), & \mathrm{x} \pm \frac{h e}{2} \in \mathrm{T}, \\
0, & \text { otherwise, }\end{cases}
\end{aligned}
$$

$h>0, \mathrm{e}_{\mathrm{i}}=(0,0, \ldots, 1,0, \ldots, 0)$ is the unit vector in $\mathbb{R}^{d}, \mathrm{e}_{\mathrm{ij}}=\mathrm{e}_{\mathrm{i}}-\mathrm{e}_{\mathrm{j}}, \mathrm{e} \in \mathbb{R}^{n} . K_{\varphi}^{\prime \prime}, 2\left(f ; t_{l}^{2}\right)_{p}$ is another K-functional of Ditzian-Totik type defined by

$$
K_{\varphi}^{*, 2}\left(f ; t_{l}^{2}\right)_{p}=\inf _{g \in W_{\Phi, p}^{2}}\left\{\|f-g\|_{p}+t_{l}^{2} \sum_{1 \leq i \leq j \leq d}\left\|\varphi_{i j}^{2} D_{i j}^{2} g\right\|_{p}\right\}, \quad t_{l}>0, l=1,2, \ldots, d .
$$

We notice that [6] for $f \in L_{p}$, we have

$$
\left\|M_{n, d}(f)-f\right\|_{p} \leq M\left(\omega_{\varphi}^{2}(f ; \sqrt{n})_{p}+n^{-1}\|f\|_{p}\right) .
$$

Thus, for $g \in W_{\Phi, p}^{2}$, by the definition of $K$-functional $K_{\varphi}^{*, 2}\left(f ; t_{l}^{2}\right)_{p}$, we have

$$
\begin{aligned}
\left\|M_{n, d}(f)-f\right\|_{p} & \leq M\left(\omega_{\varphi}^{2}(f ; \sqrt{n})_{p}+n^{-1}\|f\|_{p}\right) \\
& \leq M\left(K_{\varphi}^{*, 2}\left(f ; n^{-\frac{1}{2}}\right)+n^{-1}\|f-g\|_{p}+n^{-1}\|g\|_{p}\right) \\
& \leq M\left(2\|f-g\|_{p}+n^{-1}\|g\|_{p}+n^{-1} \sum_{1 \leq i \leq j \leq d}\left\|\varphi_{i j}^{2} D_{i j}^{2} g\right\|_{p}\right) .
\end{aligned}
$$

According to the definition of K-functional $K_{\varphi}^{2}\left(f ; t_{l}^{2}\right)_{p}$, Lemma 2.3 has been proved. 
Lemma 2.4 Suppose $1 \leq p<\infty, f \in L_{p}, n \in \mathbb{N}, n>2$. Then

$$
\Phi\left(M_{n, d}(f)\right)_{p} \leq M n K_{\varphi}^{2}\left(f ; n^{-1}\right)_{p} .
$$

Proof For $f \in L_{p}, g \in W_{\Phi, p}^{2}$, by Lemma 2.1 and Lemma 2.2, we get

$$
\begin{aligned}
\Phi\left(M_{n, d}(f)\right)_{p}= & \left\|M_{n, d}(f)\right\|_{p}+\sum_{1 \leq i \leq j \leq d}\left\|\varphi_{i j}^{2} D_{i j}^{2} M_{n, d}(f)\right\|_{p} \\
\leq & \left\|M_{n, d}(f-g)\right\|_{p}+\left\|M_{n, d}(g)\right\|_{p} \\
& +\sum_{1 \leq i \leq j \leq d}\left\|\varphi_{i j}^{2} D_{i j}^{2} M_{n, d}(f-g)\right\|_{p}+\sum_{1 \leq i \leq j \leq d}\left\|\varphi_{i j}^{2} D_{i j}^{2} M_{n, d}(g)\right\|_{p} \\
\leq & M\left(n\|f-g\|_{p}+\|g\|_{p}+\sum_{1 \leq i \leq j \leq d}\left\|\varphi_{i j}^{2} D_{i j}^{2} g\right\|_{p}\right) \\
\leq & M n\left(\|f-g\|_{p}+n^{-1}\left(\|g\|_{p}+\sum_{1 \leq i \leq j \leq d}\left\|\varphi_{i j}^{2} D_{i j}^{2} g\right\|_{p}\right)\right) .
\end{aligned}
$$

According to the definition of K-functional $K_{\varphi}^{2}\left(f ; t_{l}^{2}\right)_{p}$, Lemma 2.4 has been proved.

\section{Main results}

In this section we will prove our main results.

Theorem 3.1 Let $1 \leq p, q<\infty, n \in \mathbb{N}, n>2>\theta_{l}>0, l=1,2, \ldots, d$. Then

$$
\begin{aligned}
f \in B_{p, q}^{\frac{\theta}{2}} & \Leftrightarrow\left\{\sum_{n=1}^{\infty}\left(n^{\frac{\theta_{l}}{2}}\left\|M_{n, d}(f)-f\right\|_{p}\right)^{q} \frac{1}{n}\right\}^{\frac{1}{q}}<\infty \\
& \Leftrightarrow n^{-\frac{1}{q}} n^{\frac{\theta_{l}}{2}}\left(M_{n, d}(f ; \mathrm{x})-f(\mathrm{x})\right) \in l^{q}\left(L_{p}\right) .
\end{aligned}
$$

Proof First we prove the direct result of (3.1). By applying Lemma 2.3, we have

$$
\begin{aligned}
\sum_{n=1}^{\infty}\left[n^{\frac{\theta_{l}}{2}}\left\|M_{n, d}(f)-f\right\|_{p}\right]^{q} \frac{1}{n} & \leq \sum_{r=0}^{\infty} \sum_{n=2^{r}}^{2^{r+1}-1}\left[n^{\frac{\theta_{l}}{2}} M K_{\varphi}^{2}\left(f ; n^{-1}\right)_{p}\right]^{q} n^{-1} \\
& \leq M \sum_{r=0}^{\infty}\left[n^{(r+1) \frac{\theta_{l}}{2}} K_{\varphi}^{2}\left(f ; 2^{-r}\right)_{p}\right]^{q} \\
& \leq M \frac{1}{\ln 2}\left(2^{1+\frac{\theta_{l}}{2}}\right)^{q} \sum_{r=0}^{\infty} \int_{2^{-(r+1)}}^{2^{-r}}\left[t^{-\frac{\theta_{l}}{2}} K_{\varphi}^{2}(f ; t)_{p}\right]^{q} \frac{d t}{t} \\
& \leq M \frac{1}{\ln 2}\left(2^{1+\frac{\theta_{l}}{2}}\right)^{q} \int_{0}^{1}\left[t^{-\frac{\theta_{l}}{2}} K_{\varphi}^{2}(f ; t)_{p}\right]^{q} \frac{d t}{t} .
\end{aligned}
$$

In virtue of $f \in B_{p, q}^{\frac{\theta}{2}}$ and by Theorem 1.2, we have

$$
\sum_{n=1}^{\infty}\left[n^{\frac{\theta_{l}}{2}}\left\|M_{n, d}(f)-f\right\|_{p}\right]^{q} \frac{1}{n}<\infty
$$

The necessity has been proved. 
Next, we prove the inverse result of (3.1). We take a constant $A \in \mathbb{N}$, which will be determined later. For $r \in \mathbb{N}$, we take $n_{r} \in \mathbb{N}$, which satisfies the following conditions:

$$
\text { (1) } A^{r-1} \leq n_{r}<A^{r} ; \quad \text { (2) }\left\|M_{n_{r}, d}(f)-f\right\|_{p}=\min _{A^{r-1} \leq m<A^{r}}\left\|M_{m, d}(f)-f\right\|_{p} \text {. }
$$

By using the definition of K-functional and Lemma 2.4, we derive by induction

$$
\begin{aligned}
A^{\frac{\theta}{2}} K_{\varphi}^{2}\left(f ; A^{-r}\right)_{p} \leq & A^{\frac{\theta_{l}}{2}}\left\|f-M_{n_{r}, d}(f)\right\|_{p}+M A^{\left(\frac{\theta_{l}}{2}-r\right)} n_{r} K_{\varphi}^{2}\left(f ; n_{r}^{-1}\right)_{p} \\
\leq & A^{\frac{\theta_{l}}{2}}\left\|f-M_{n_{r}, d}(f)\right\|_{p}+A^{r\left(\frac{\theta_{l}}{2}-1\right)}\left[M n_{r}\left\|f-M_{n_{r-1}, d}(f)\right\|_{p}\right. \\
& \left.+M^{2} n_{r-1} K_{\varphi}^{2}\left(f ; n_{r-1}^{-1}\right)_{p}\right] \\
\leq & \cdots \\
\leq & A^{\frac{\theta_{l}}{2}}\left\|f-M_{n_{r}, d}(f)\right\|_{p}+A^{r\left(\frac{\theta_{l}}{2}-1\right)}\left[\sum_{v=1}^{r-1} M^{l} n_{r-v+1}\left\|f-M_{n_{r-v}, d}(f)\right\|_{p}\right. \\
& \left.+M^{r} n_{1} K_{\varphi}^{2}\left(f ; n_{1}^{-1}\right)_{p}\right] \\
\leq & A^{1+\frac{\theta_{l}}{2}} \sum_{v=1}^{r-1}\left(M A^{v\left(\frac{\theta_{l}}{2}-1\right)}\right)^{v}\left[n_{r-v}^{\frac{\theta_{l}}{2}}\left\|f-M_{n_{r-v}, d}(f)\right\|_{p}\right] \\
& +A\left(M A^{\frac{\theta_{l}}{2}-1}\right)^{r}\|f\|_{p} .
\end{aligned}
$$

We now choose $A \in \mathbb{N}, A \geq 2$, such that $\alpha:=M A^{\frac{\theta_{l}}{2}-1}<\frac{1}{2}$. For $1<q<\infty$, we have

$$
\begin{aligned}
& \int_{0}^{A^{-1}}\left[t_{l}^{-\frac{\theta_{l}}{2}} K_{\varphi}^{2}\left(f ; t_{l}\right)_{p}\right]^{q} \frac{d t_{l}}{t_{l}} \\
& \leq A^{\frac{\theta_{l} q}{2}} \ln A \sum_{r=0}^{\infty}\left[A^{\frac{k \theta_{l}}{2}} K_{\varphi}^{2}\left(f ; A^{-r}\right)_{p}\right]^{q} \\
& \leq 2^{q} A^{\frac{\theta_{l} q}{2}}(\ln A) A^{\left(1+\frac{\theta_{l}}{2}\right) q} \sum_{r=1}^{\infty}\left\{\left[\sum_{v=0}^{r-1} \alpha^{v} n_{r-1}^{\frac{\theta_{l}}{2}}\left\|f-M_{n_{r-v}, d}(f)\right\|_{p}\right]^{q}+A^{q}\left(\alpha^{v}\|f\|_{p}\right)^{q}\right\} \\
& \leq A_{1} A^{q} \frac{\alpha}{1-\alpha}\|f\|_{p}^{q}+2^{q-1} A_{1} \sum_{\nu=1}^{\infty} \sum_{r=v+1}^{\infty} \alpha^{r-v}\left[n_{v}^{\frac{\theta_{l}}{2}}\left\|f-M_{n_{v}, d}(f)\right\|_{p}\right]^{q} \\
& \leq 2 A_{1} A^{q}\|f\|_{p}^{q}+2^{q-1} A_{1} \sum_{v=1}^{\infty}\left[n_{v}^{\frac{\theta_{l}}{2}}\left\|f-M_{n_{v}, d}(f)\right\|_{p}\right]^{q} \\
& \leq M\left\{\|f\|_{p}^{q}+\sum_{v=1}^{\infty} \sum_{A^{v-1} \leq m<A^{v}}\left[m^{\frac{\theta_{l}}{2}}\left\|f-M_{m, d}(f)\right\|_{p}\right]^{q}\right\}<\infty .
\end{aligned}
$$

The proof for $q=1$ is easy and we shall omit it. Thus, we have

$$
\int_{0}^{1}\left[t_{l}^{-\frac{\theta_{l}}{2}} K_{\varphi}^{2}\left(f ; t_{l}\right)_{p}\right]^{q} \frac{d t_{l}}{t_{l}}<\infty .
$$

By Theorem 1.2, the sufficiency has also been proved. The proof is completed. 
Remark 1 For other integral-type operators, the method and the results are similar.

\section{Competing interests}

The authors did not provide this information.

\section{Authors' contributions}

The authors did not provide this information.

\section{Author details}

${ }^{1}$ School of Mathematics and Information Engineering, Taizhou University, Taizhou, Zhejiang 317000, China. ${ }^{2}$ School of Mathematical Sciences, Beijing Normal University, Beijing, 100875, China. ${ }^{3}$ School of Science, China University of Mining and Technology, Beijing, 100083, China.

\section{Acknowledgements}

The authors would like to thank the anonymous referees for their valuable comments, remarks and suggestions which greatly helped us to improve the presentation of this paper and make it more readable. Project supported by the Natural Science Foundation of China (Grant No. 10671019), the Zhejiang Provincial Natural Science Foundation (Grant No. LY12A01008), and the Cultivation fund of Taizhou University.

\section{Received: 15 July 2011 Accepted: 10 April 2013 Published: 23 April 2013}

\section{References}

1. Derriennic, MM: On multivariate approximation by Bernstein-type polynomials. J. Approx. Theory 45, 155-166 (1985)

2. Nikolskii, SM: Approximation of Functions of Several Variables and Imbedding Theorem. Springer, Berlin (1975)

3. Bergh, J, Lörfstrom, J: Interpolation Spaces. Springer, Berlin (1976)

4. Cao, FL, Xiong, JY: Stechkin-Marchaud type inequalities in connection with $L_{p}$ approximation for multivariate Bernstein-Durrmeyer operators. Chin. Ann. Math. 22(2), 151-156 (2001)

5. Berens, $H, X u, Y: K$-moduli of smoothness, and Bernstein polynomials on a simplex. Indag. Math. 2(4), 411-421 (1991)

6. Berens, H, Schmid, HJ, Xu, Y: Bernstein-Durrmeyer operators on a simplex. J. Approx. Theory 68, 247-261 (1992)

\section{Submit your manuscript to a SpringerOpen ${ }^{\circ}$ journal and benefit from:}

- Convenient online submission

- Rigorous peer review

- Immediate publication on acceptance

- Open access: articles freely available online

- High visibility within the field

- Retaining the copyright to your article 